\title{
The divergent protective effects of angiotensin-converting enzyme inhibitors and angiotensin receptor blockers on clinical outcomes of coronavirus disease 2019 (COVID-19): a systematic review and meta-analysis
}

\author{
Qiuping Xie ${ }^{1}$, Shuai Tang ${ }^{2}$, Yining $\mathrm{Li}^{3}$ \\ ${ }^{1}$ Zhuzhou Central Hospital, Department of Cardiology, Zhuzhou, China; ${ }^{2}$ Xuzhou Medical University, Department of Pharmacy, Xuzhou, China; \\ ${ }^{3}$ Second Xiangya Hospital, Department of Radiology, Changsha, China \\ Contributions: (I) Conception and design: Q Xie, Y Li; (II) Administrative support: None; (III) Provision of study materials or patients: None; \\ (IV) Collection and assembly of data: S Tang, Y Li; (V) Data analysis and interpretation: Q Xie, S Tang; (VI) Manuscript writing: All authors; (VII) \\ Final approval of manuscript: All authors. \\ Correspondence to: Qiuping Xie. Department of Cardiology, Zhuzhou Central Hospital, Zhuzhou 412007, China. Email: xieqiuping@126.com; \\ Yining Li. Department of Radiology, Second Xiangya Hospital, Changsha 410011, China. Email: xiangyaliyining@csu.edu.cn or 664009149@qq.com.
}

Background Some studies have speculated that patients on angiotensin-converting enzyme inhibitors (ACEIs) or angiotensin receptor blockers (ARBs) are more susceptible to adverse outcomes of coronavirus disease 2019 (COVID-19). Here, we performed a systematic review and meta-analysis to evaluate the safety and efficacy of administering ACEIs and ARBs to patients with COVID-19.

Methods: Studies of COVID-19 were collected from the PubMed, Embase, medRxiv and BioRxiv databases. The pooled relative risk odds ratio (OR) and 95\% confidence interval (95\% CI) were calculated. Subgroup analyses were conducted by medication (ACEIs and ARBs) and geographical location (China and outside China). Inter-study heterogeneity was assessed using meta-regression. Begg's test, Egger's test and funnel plots were adopted to evaluate possible publication bias.

Results: Thirty studies containing 10,434 adult patients were included in our meta-analysis. The pooled result indicated that the administration of ACEIs or ARBs reduced the risk of severe/death outcomes for COVID-19 patients. Meanwhile, a significant reduction in the risk of severe/death outcomes was observed to be associated with the administration of ACEIs or ARBs among COVID-19 patients in China, but this association was weaker for studies outside China. Furthermore, ACEI therapy was found to carry a significantly lower risk of an adverse clinical outcome.

Discussion: Our systematic review and meta-analysis found that neither ACEIs nor ARBs worsen the clinical outcomes of COVID-19 patients. On the contrary, we found that patients treated with ACEIs or ARBs have a reduced risk of severe/death outcomes, especially in Asia. Furthermore, ACEIs may reduce the risk of severe/death outcomes. Therefore, treatment interruption of ACEI or ARB therapy during COVID-19 infection is not recommended.

Keywords: Coronavirus disease 2019 (COVID-19); angiotensin-converting enzyme inhibitors (ACEIs); angiotensin receptor blockers (ARBs); clinical outcomes; meta-analysis

Submitted Apr 21, 2021. Accepted for publication Sep 30, 2021.

doi: 10.21037/apm-21-972

View this article at: https://dx.doi.org/10.21037/apm-21-972 


\section{Introduction}

Severe acute respiratory syndrome coronavirus 2 (SARS-CoV-2) first presented as an outbreak of atypical pneumonia in Wuhan, China, on December 12, 2019 (1). Since then, the virus has spread, and as of May 1, 2020, it had caused 3,175,207 infections and claimed 224,172 lives in over 200 countries.

SARS-CoV-2 infection disproportionately affects older people with hypertension, diabetes mellitus, and cardiovascular disease, and patients with these comorbidities are often treated with renin-angiotensin-aldosterone system (RAAS) inhibitors, including angiotensin-converting enzyme inhibitors (ACEIs) and angiotensin receptor blockers (ARBs) $(2,3)$.

Antihypertensive drugs, including RAAS inhibitors, have been reported to increase the levels of angiotensinconverting enzyme 2 (ACE2), which is a functional receptor for SARS-CoV-2 (4-6) (Figure 1). These observations have prompted concern that the administration of RAAS inhibitors may facilitate coronavirus disease 2019 (COVID-19) infection and worsen the prognosis. Recently, two large, well-conducted studies found that previous treatment with RAAS blockers does not raise the risk of onset or aggravation (including death) of COVID-19 $(7,8)$. Controversially, other studies have reported that COVID-19 patients who received ACEI/ARB therapy were less susceptible to adverse outcomes (9-11).

Given the worldwide use of ACEIs and ARBs and the inconsistency of clinical research results, a comprehensive evaluation of the relationship between ACEIs and ARBs and the clinical outcomes of patients with COVID-19 is urgently needed. Here, we conducted a meta-analysis to comprehensively and quantitatively evaluate the safety and effectiveness of RAAS inhibitor administration in older patients with COVID-19. We present the following article in accordance with the PRISMA and MOOSE reporting checklists (available at https://apm.amegroups.com/article/

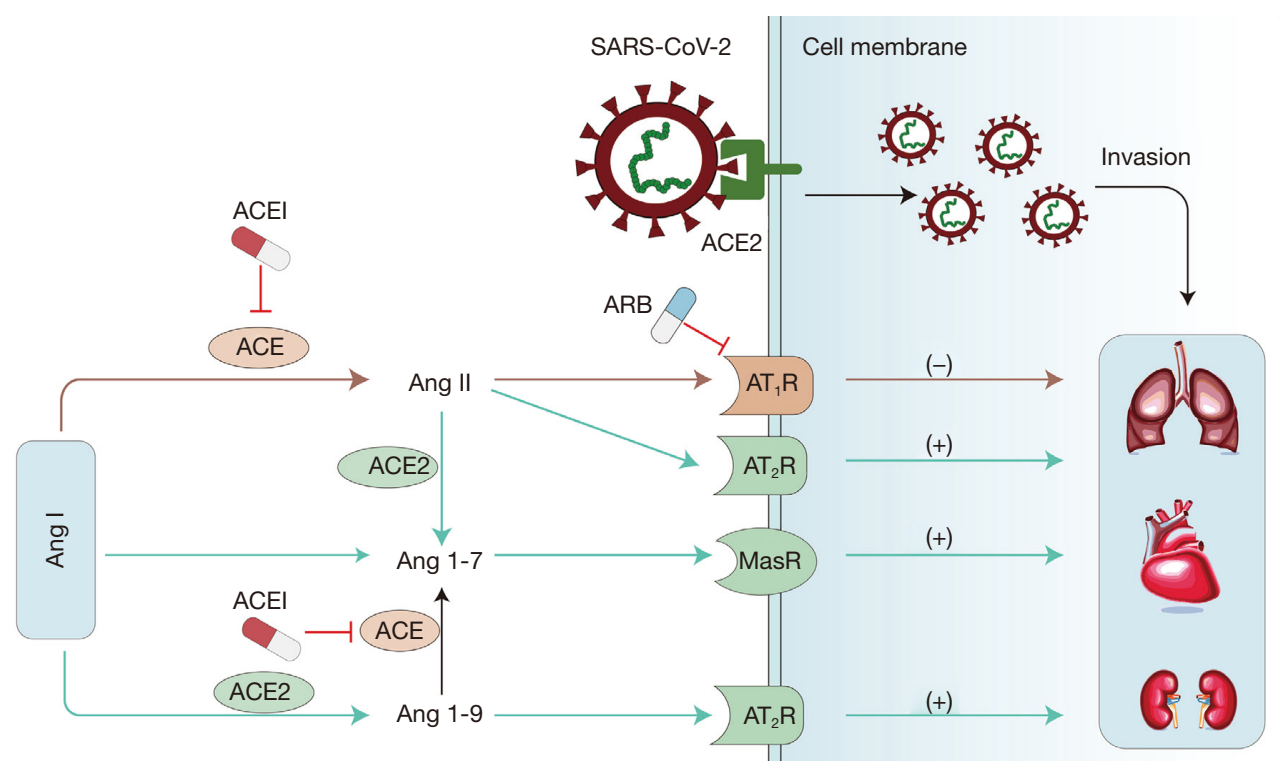

Figure 1 Schematic diagram of the RAS showing the role of ACE2 as a key element in the counter-regulatory axis of the RAS (elements in green). ACE2 opposes the harmful effects of the Ang II-AT ${ }_{1} \mathrm{R}$ axis (elements in brown) on injury by activating MasR and $\mathrm{AT}_{2} \mathrm{R}$ signaling. After infection, SARS-CoV-2 binds through its viral spike protein to host cell membrane-bound ACE2, thereby promoting viral cell entry and subsequent replication. Importantly, the binding of SARS-CoV-2 may lead to the downregulation of ACE2. Impairment of ACE2 activity results in the activation of the harmful Ang II-AT $\mathrm{R}$ axis, which aggravates the viral pathogenicity of SARS-CoV-2, tipping the scale in favor of lung, heart, and kidney damage. ARBs have been shown to increase ACE2 expression in various tissues. Treatment with ACEIs primarily protects against lung injury by reducing Ang II levels through the inhibition of Ang I to Ang II conversion. (+), protection; (-), injury. ACEI, angiotensin-converting enzyme inhibitor; ARB, angiotensin receptor blocker; ACE, angiotensin-converting enzyme; ACE2, angiotensin-converting enzyme 2; $\mathrm{AT}_{1} \mathrm{R}$, angiotensin II receptor type 1; $\mathrm{AT}_{2} \mathrm{R}$, angiotensin II receptor type 2; MasR, Mas receptor; SARS$\mathrm{CoV}-2$, severe acute respiratory syndrome coronavirus 2; RAS, renin-angiotensin system. 

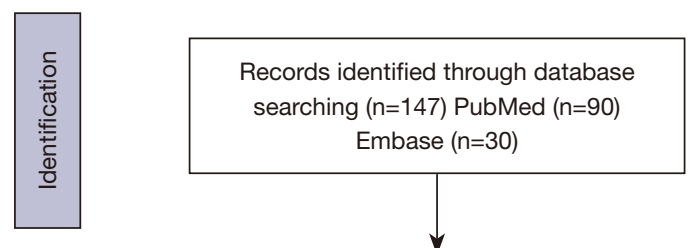

Additional records identified through other sources medRxiv and Embase $(n=30)$ bioRxiv $(n=27)$
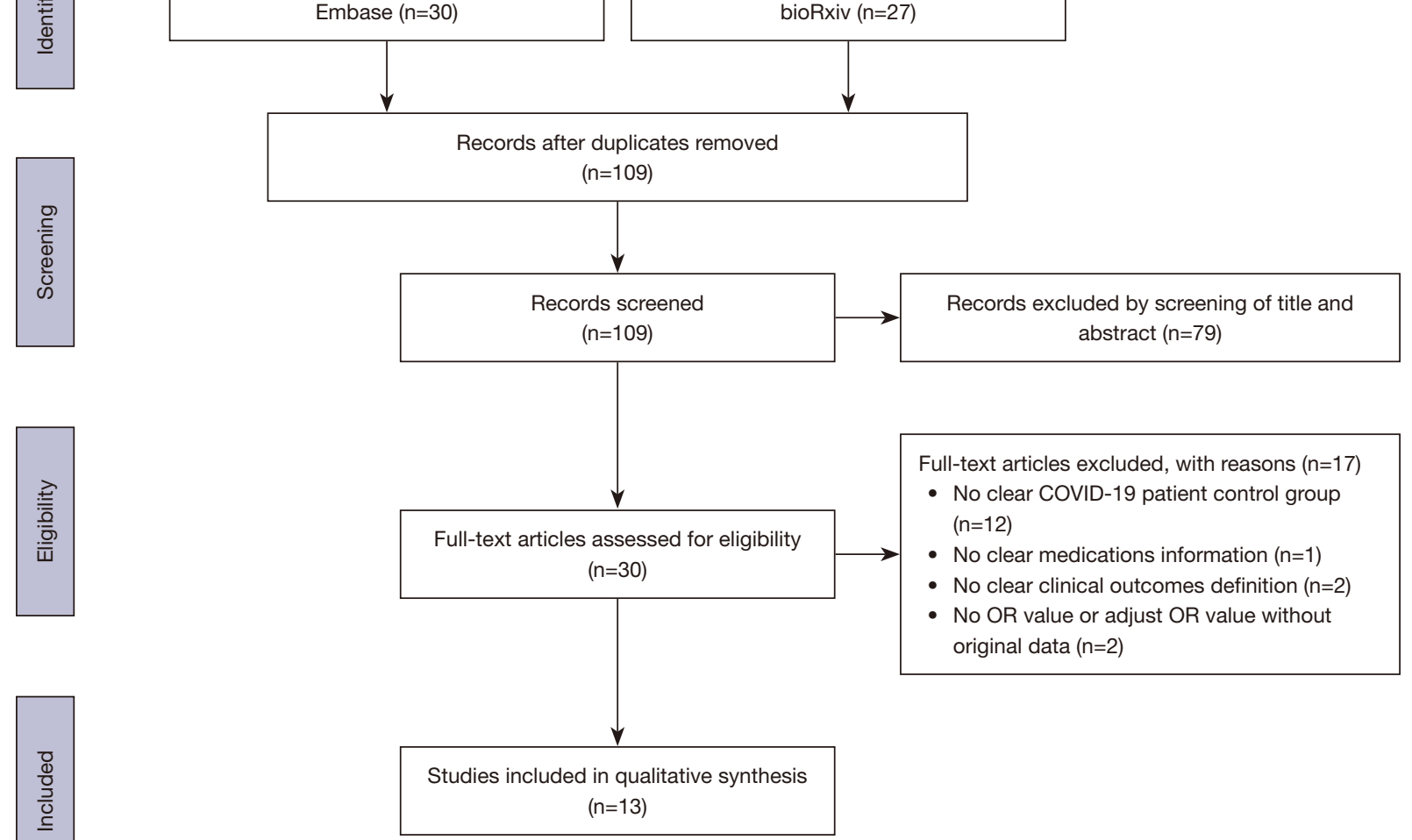

Full-text articles excluded, with reasons $(n=17)$

- No clear COVID-19 patient control group $(n=12)$

- No clear medications information $(n=1)$

- No clear clinical outcomes definition $(n=2)$

- No OR value or adjust OR value without original data $(n=2)$

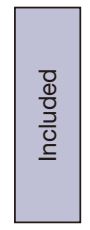

Figure 2 Flowchart depicting the literature search and selection strategy. After applying the inclusion and exclusion criteria, a total of 13 articles were included in the final meta-analysis. COVID-19, coronavirus disease 2019; OR, odds ratio.

view/10.21037/apm-21-972/rc).

\section{Methods}

\section{Search strategy}

Articles from online databases (PubMed and EMBASE) were searched using specific strategies introduced in Appendix. Preprint articles from medRxiv and BioRxiv were retrieved using the following keywords: COVID19, 2019 novel coronavirus disease, COVID-19 pandemic, SARSCoV-2 infection, OR COVID-19 virus disease, 2019 novel coronavirus infection, 2019-nCoV infection, 2019-nCoV infection, COVID-19, 2019-nCoV disease, COVID-19 virus infection, and renin-angiotensin-aldosterone system, RAAS, angiotensin-converting enzyme inhibitors, ACEIs, angiotensin II receptor blockers, ARBs. The reference lists of the review articles and selected articles were manually searched to identify additional relevant studies. Studies published between January 01, 2020, and May 05, 2020, were included in our study. No patients were involved in this study.

\section{Eligibility criteria}

Studies evaluating the effects of RAAS inhibitor (ACEIs or ARBs) therapy on the clinical outcomes of patients with COVID-19 (severe disease or death during hospitalization) were enrolled (Figure 2). Clinical trials, such as those with a randomized or non-randomized, parallel-group, or cluster design, and clinical observational studies, including retrospective or prospective cohort studies and case-control studies, that reported on disease severity/death outcomes and the use of RAAS in patients with COVID-19 were also included in our analysis. Letters or comments with effective control groups were also included. Studies with peer-reviewed and preprint articles were included without language restrictions. Letters or comments, review articles, 
case reports, and any articles without effective controls were excluded, as were articles with overlapping samples.

\section{Data selection and extraction}

The titles and abstracts of the retrieved articles were independently evaluated by two authors (QX and YL). Articles with titles and abstracts meeting the eligibility criteria were downloaded for further data selection and extraction. Two authors independently read and extracted data from the selected studies in duplicate. Any discordance in data was resolved by the third author (ST). Endnote (X9) was used to manage citations and data extraction. The following data were extracted for the meta-analysis: first authors, year of publication, country of recruitment, study design, type(s) of RAAS inhibitor, clinical outcomes (defined in each study), the number of events, and total cases in each group, crude odds ratio (cOR) value [95\% confidence interval (CI)], adjusted OR (aOR) value (95\% CI) (if available), and adjusted factors (Table 1).

\section{Risk of bias assessment}

The study quality was assessed using a qualitative classification method to evaluate the risk of bias (high, moderate or low) (Table S1). Reports were defined as having a low risk of bias if adjustment for both age and sex was reported. Studies reporting adjustment for age or sex were defined as having a moderate risk of bias, while those with no adjustment were classified as high risk. Publication bias was evaluated using Begg's and Egger's asymmetry tests $(12,13)$ and was presented as a funnel plot.

\section{Statistical analysis}

In this study, the extracted aOR and cOR values were used to calculate pooled ORs with $95 \%$ CIs. I ${ }^{2}$ statistics were used to appraise inter-study heterogeneity, with $\mathrm{I}^{2} \geq 50 \%$ defined as significant heterogeneity. In cases of $\mathrm{I}^{2}<50 \%$, a fixed effects model was used to calculate the pooled OR and $95 \%$ CI, and if $\mathrm{I}^{2} \geq 50 \%$, a random effects model was used (13). Firstly, we analyzed the effects of primary RAAS inhibitors on severe/death outcomes in patients with COVID-19. Secondly, subgroup analyses were conducted by medication (ACEIs and ARBs) and geographical location (China and outside China). Sensitivity analyses were conducted by (I) deleting each eligible study, in turn, to assess whether one study dominated the results of the meta-analysis; (II) eliminating low-quality studies. Inter-study heterogeneity was calculated by meta-regression analysis. Stata software (Version 15.0) was used to perform all statistical analyses.

\section{Results}

\section{Descriptions of the included studies}

Our meta-analysis included 33 studies involving 10,434 patients, with 4,414 individuals in the RAAS inhibitor group [including 833 patients (18.87\%) with severe/death outcomes] and 6,020 patients in the control group [including 1,134 patients $(18.83 \%)$ with severe/death outcomes] (Table 1 ) (7-11,14). Besides, all 13 studies had a retrospective design, with 5 studies included from medRxiv $(9,11,14-16)$ and 8 from peer-reviewed databases $(7,10,17-20)$. There were 9 studies from Asia (China) (11,14-21), 2 studies from Europe [from Italy (8) and the UK (9)], and 1 from North America (USA) (7), while 1 study included COVID-19 patients from Asia, Europe and North America (10). Details of the study characteristics are presented in Table 1.

\section{$R A A S$ inhibitors were associated with a significantly reduced risk of severe/death outcomes in patients with COVID-19}

In the 13 studies, 7 reported the aOR values comparing severe/death outcomes in COVID-19 patients on RAAS inhibitors, with inconsistent results $(8-11,15,16,21)$. Of these studies, Bean et al. and Mehra et al. $(9,10)$ reported that patients treated with ACEIs had a significantly reduced risk of severe/death outcomes than those who did not receive ACEIs. Feng et al. and Zhang et al. $(11,16)$ reported that ACEI/ARB therapy had the significant effect of reducing the occurrence of severe/death outcomes. However, Mehra et al. did not report the same results for ARBs. The studies of Yan et al., Mancia et al. and Feng et al. $(8,11,15)$ revealed no significant effects of ACEI/ARB therapy on severe/death outcomes in patients with COVID-19.

In a pool of the 7 studies, we found that RAAS inhibitors reduced the risk of COVID-19 patients developing to severe/death outcomes (overall OR $=0.79$; 95\% CI: $0.63-$ $0.98 ; \mathrm{P}<0.0001)$, with significant heterogeneity between the included studies $\left(\mathrm{I}^{2}=54.6 \%\right)$ (Figure 3) $(8-11,15,16,21)$. The risk of bias analysis showed that 5 of the 7 adjusted articles were low risk $(8,9,15,16,21)$, while 2 were moderate risk $(10,11)$ (Table S1). The sensitivity analyses revealed no significant differences from the primary analysis (Table S2), 


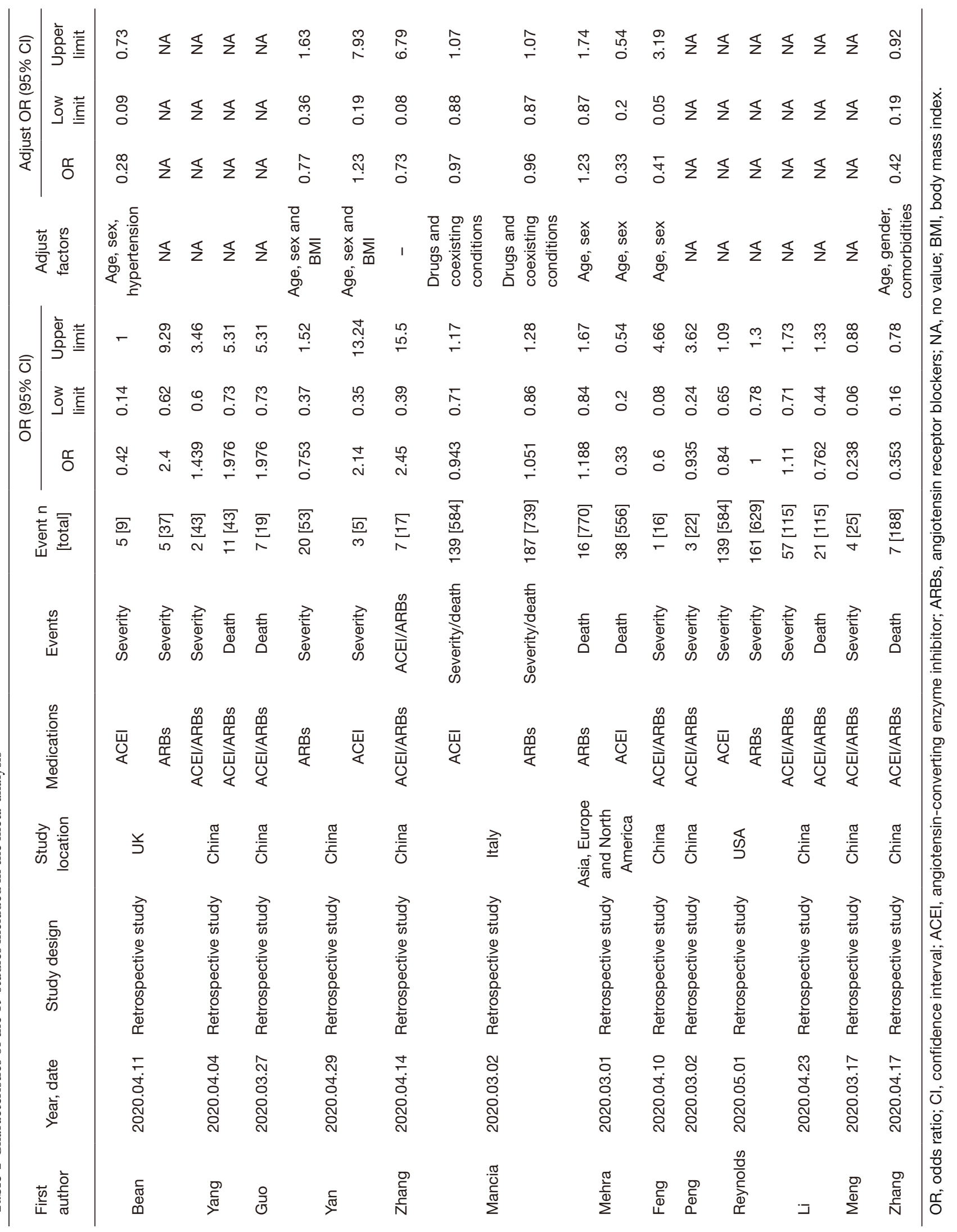




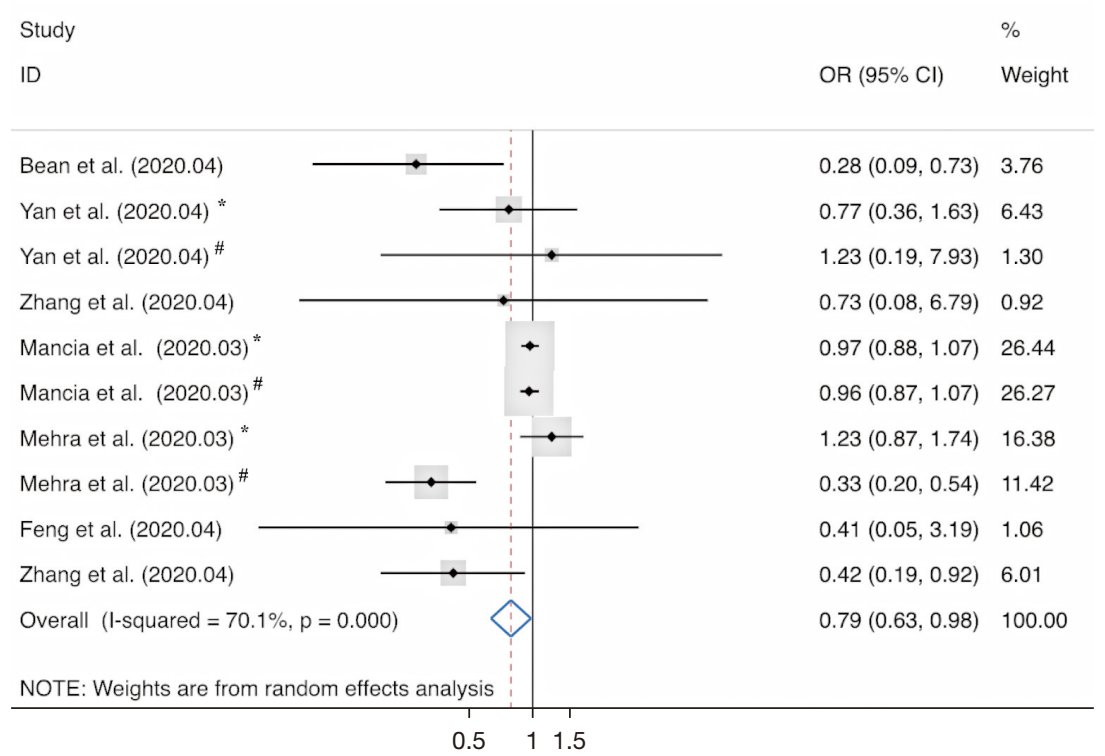

Figure 3 Forest plot showing the effect of RAAS therapy on the risk of adverse clinical outcomes in patients with COVID-19. In this and subsequent figures, the horizontal lines indicate the lower and upper limits of the $95 \%$ CI. *, COVID-19 patients with ACEI therapy; *, COVID-19 patients with ARB therapy. OR, odds ratio; CI, confidence interval; RAAS, renin-angiotensin-aldosterone system; COVID-19, coronavirus disease 2019; ACEI, angiotensin-converting enzyme inhibitor; ARB, angiotensin receptor blocker.

showing the results were reliable. However, no significant reducing effect was observed when the $\mathrm{cOR}$ values were pooled (overall OR $=0.93 ; 95 \%$ CI: $0.84-1.02 ; \mathrm{P}=0.001$; $\mathrm{I}^{2}=56.4 \%$ ) (Figure $\mathrm{S} 1$ ).

Of the 7 studies included, 4 studies were conducted in China and reported the aOR values $(11,15,16,21)$. Zhang et al.'s study reported that ACEI/ARB therapy reduces the effect on patients with COVID-19 (HR $=0.42$; 95\% CI: 0.19-0.92), whereas the other 3 studies did not report the same result. However, in our subgroup analyses, we divided these 4 studies into a China group with aOR values by random effects model, and the results showed a significant reducing effect in this group (overall OR $=0.60 ; 95 \%$ CI: $0.37-0.99 ; \mathrm{P}=0.75)$ with no heterogeneity $\left(\mathrm{I}^{2}=0.0 \%\right)$ (Figure 4$)$. This result was coincident with those of fixed effects analyses (overall OR $=0.60 ; 95 \% \mathrm{CI}$ : 0.37-0.99; $\mathrm{P}=0.75$ ) (Figure S2).

\section{ACEIs may be effective in reducing the risk of severe/death outcomes among patients with COVID-19}

In the preliminary analysis, no significant effect was observed in the subgroup analysis restricted to COVID-19 patients treated with ACEIs/ARBs (8 studies) (11,14,16-21), ACEIs (5 studies) and ARBs (5 studies) (7-10,15) (data not shown). We further combined data from the ACEI/ARB subgroup into subgroups exclusively, including ACEIs and ARBs, respectively, to obtain more robust results. No significant reducing effect was observed based on the random effects model using cOR values (overall $\mathrm{OR}=0.76$; 95\% CI: 0.58-0.99; $\mathrm{P}<0.002 ; \mathrm{I}^{2}=56.7 \%$ ) (Figure S3). However, as shown in Figure 5, the benefits of ACEI and ACEI/ARB treatment on reducing the risk of severe/death outcomes were further observed depending on the a OR values (overall $\mathrm{OR}=0.52 ; 95 \% \mathrm{CI}$ : $0.28-0.96 ; \mathrm{P}<0.0001$, $\left.\mathrm{I}^{2}=77.7 \%\right)$. In contrast, no significant effect was observed when the ACEI/ARB group was combined with the ARBs group (overall $\mathrm{OR}=0.96 ; 95 \% \mathrm{CI}$ : 0.87-1.06; $\mathrm{P}=0.205$; $\mathrm{I}^{2}=30.7 \%$ ) (Figure S4).

Meanwhile, subgroup analysis by China and outside China using aOR values were also performed. A reducing effect was observed in the China group (overall $\mathrm{OR}=0.50$; 95\% CI: $0.26-0.97 ; \mathrm{P}<0.747 ; \mathrm{I}^{2}=0.0 \%$ ) by fixed effects analysis (Figure S5).

\section{Heterogeneity and publication bias}

Significant heterogeneity was found in the pooled metaanalysis to estimate the association of medications and the occurrence of severe/death outcomes, with an $\mathrm{I}^{2}$ of $70.1 \%$ 


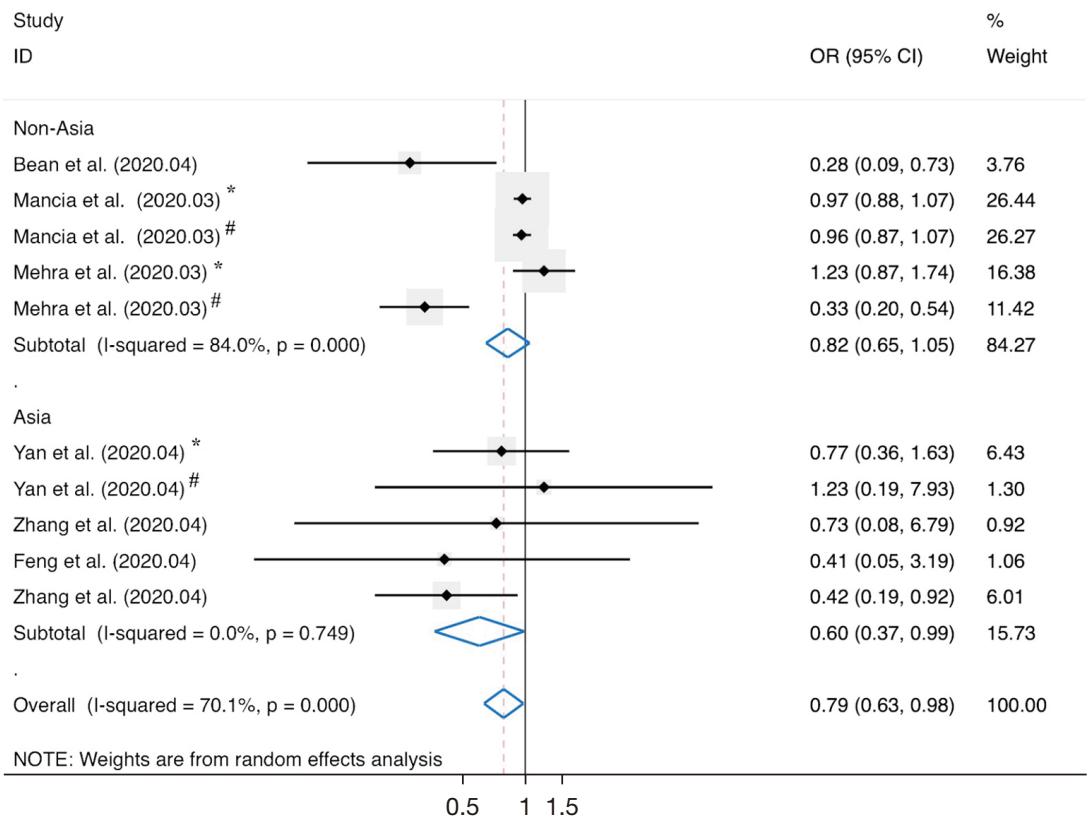

Figure 4 Forest plot showing the effect of different study locations on the risk of adverse clinical outcomes in patients with COVID-19. *, COVID-19 patients with ACEI therapy; ", COVID-19 patients with ARB therapy. OR, odds ratio; CI, confidence interval; COVID-19, coronavirus disease 2019; ACEI, angiotensin-converting enzyme inhibitor; ARB, angiotensin receptor blocker.

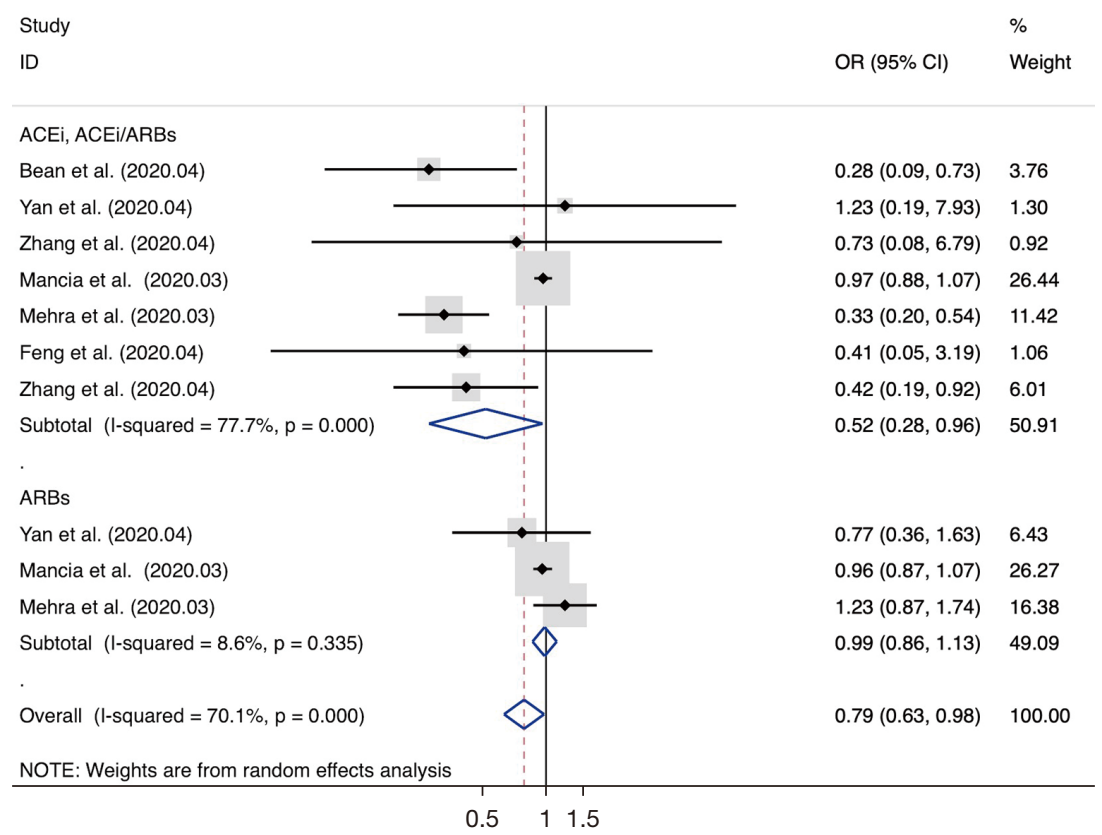

Figure 5 Forest plot showing the effect of different medications on the risk of adverse clinical outcomes in patients with COVID-19. OR, odds ratio; CI, confidence interval; ACEI, angiotensin-converting enzyme inhibitor; ARBs, angiotensin receptor blockers; COVID-19, coronavirus disease 2019. 


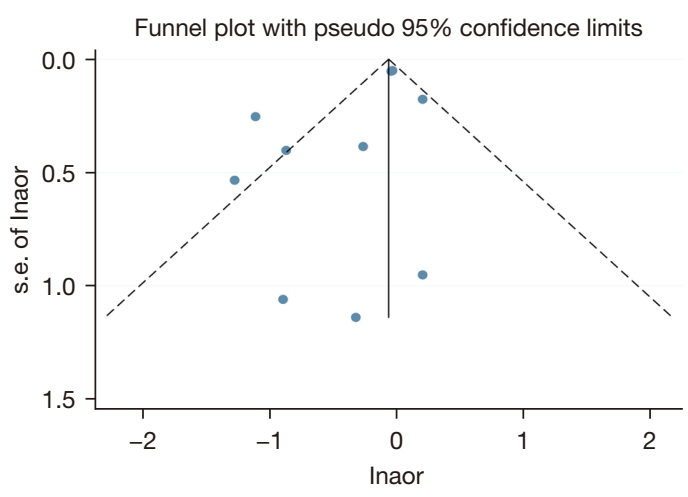

Figure 6 Funnel plot for the publication bias in the overall analysis.

$(\mathrm{P}<0.000)$. According to the meta-regression analyses based on factors including medications, clinical outcomes, journal, study location, and total case number, no variables were statistically significantly associated with the effects of medications and severe/death outcomes in patients with COVID-19 (Table S3).

In the overall analysis of the risk relationship between RAAS inhibitors and poor clinical outcomes in patients with COVID-19, the publication bias funnel plots were symmetrical, suggesting no evident publication bias (Figure 6). Both Begg's and Egger tests indicated no significant publication bias $(\mathrm{P}=0.833$ and $\mathrm{P}=0.822$ for $\mathrm{cOR} ; \mathrm{P}=0.324$ and $\mathrm{P}=0.325$ for aOR) (Tables $\mathrm{S} 4, \mathrm{~S} 5)$.

\section{Discussion}

Our meta-analysis of 13 eligible studies showed that neither ACEIs nor ARBs were harmful to COVID-19 outcomes. The odds of COVID-19 patients who are treated with ACEIs and/or ARBs having a severe/death clinical outcome were lower $(21 \%)$ than those of patients without RAAS inhibitor treatment. These findings are in line with those of a previous systematic review study which identified a reduced risk of pneumonia in patients treated with ACEIs/ ARBs along with an association between the use of ACEIs and a reduction in pneumonia-related mortality (22).

The mechanisms by which RAAS inhibitors reduce the risk of adverse outcomes of COVID-19 remain inconclusive. However, multiple animal studies have proved that activating the Ang II-AT $\mathrm{R}_{1}$ pathway triggered by the downregulation (or loss) of ACE2 in the RAAS system might be a contributing mechanism of SARS-CoVmediated acute lung injury, especially acute respiratory distress syndrome (23-27). Therefore, we speculate that
RAAS inhibitors might protect COVID-19 patients from severe acute lung injury in the same manner.

Meanwhile, extensive epidemiological evidence shows that COVID-19 infection can cause myocardial injury (28-32) and worsen and complicate pre-existing conditions, leading to death from cardiovascular events $(2,28)$. Given that the role of RAAS inhibitors in the pathophysiology of cardiovascular disease is well-established $(13,17)$, we hypothesized that the protective effect of RAAS inhibitors on COVID-19 outcomes might lie in their effects on both lung injury and the cardiovascular system.

Interestingly, a pooled OR calculated using the cOR values in our study failed to show any statistical correlation between the use of RAAS inhibitors and COVID-19 outcomes until we made an estimate using aOR values. Therefore, we assumed that some studies found no protective effect of RAAS inhibitors may contribute to concluding from the unadjusted results $(16,29)$.In this respect, the findings from a landmark study involving 8,910 patients may more strongly justify the role of RAAS inhibitors in COVID-19 treatment by considering a wide range of confounders, including age and sex and comorbidities (10).

In our subgroup analysis, estimates using data exclusively regarding ACEIs and ARBs, respectively, failed to obtain a significant effect, mainly due to fewer studies including data for this exclusive group. However, the effect of ACEIs obtained from the pooled data of ACEIs, and ACEIs/ ARBs might be underestimated since different medication stratification strategies obtained similar results, with COVID-19 patients on ACEIs being less susceptible to poor clinical outcomes compared with those not treated with RAAS inhibitors. Meanwhile, such a significant association was not observed in patients on ARBs. Although significant statistical heterogeneity for ACEIs existed, all estimates for study designs shared the same direction. Previous systematic meta-analyses have repeatedly demonstrated that ACEI treatment is more effective in reducing the risk of pneumonia, cardiovascular disease and death (22,30-33). In light of the above consistency, we interpreted that ACEIs might have a more favorable effect than ARBs for COVID-19 patients; however, further evidence is warranted to confirm this. Concerning the mechanism of action, bradykinin accumulation resulting from the use of ACEIs is regarded as an important and unique reason responsible for cardiovascular protection, especially in the prevention of ischemia-reperfusion injury and the improvement of endothelial function (34). 
Our analyses showed that most of the potential protective benefits of RAAS inhibitors seemed to be observed in Chinese patients. This finding was inconsistent with Grover's meta-analysis (preprint publication) regarding the effect of ACEIs/ARBs on Chinese COVID-19 patients. However, we cast doubt on the rationality of the analytical method and the reliability of the results of Grover's study since all of its analyses were based on cORs, the ACEI and $\mathrm{ARB}$ data were pooled directly, and repetitive studies were included (studies from Meng et al. and Liu et al.) (20,29,35). In agreement with our findings, the previous meta-analysis also reported that Asian patients could benefit more from treatment with ACEIs in reducing the risk of pneumonia (22). Mechanistically, the high expression of ACE2 in Asians may account for the differences in the protective effect of ACEIs, since a higher level of ACE2 is related to a favorable prognosis of COVID-19 infection (36). However, in general, our conclusions are weak, as the studies from China were retrospective observational studies with small sample sizes. It is unclear whether the methodology of the studies or the clinical and genetic characteristics of the patients were responsible for this finding.

Our meta-analysis estimated the pooled effect using aOR values to obtain more robust results; however, this study still has limitations. Firstly, we analyzed factors using subgroup and meta-regression in our study, but we could not obtain individual data to address the within-study heterogeneity. Secondly, most studies were retrospective studies or included retrospective cohorts, which limited our ability to infer the real causal relationship. Thirdly, some studies included in this meta-analysis were collected from preprint manuscripts without peer review. Finally, the studies included from Asia were all conducted in native Chinese populations, and studies from broad geographic areas should be evolved over time. Strengths and limitations of this study are as follows: (I) our systematic review and meta-analysis found that neither ACEIs nor ARBs worsen the clinical outcomes of COVID-19 patients. (II) Patients who accepted ACEI or ARB therapy had a reduced risk of severe/death outcomes, especially in Asia. (III) ACEIs may play a more effective role in lowering the risk of severe/ death outcomes in patients with COVID-19.

\section{Conclusions}

Our results suggest that neither ACEIs nor ARBs are harmful to COVID-19 outcomes, and ACEIs might have a more favorable effect than ARBs in treating COVID-19 patients with comorbidities. Therefore, interrupting ACEI/ ARB therapy to prevent severe/death from COVID-19 infection is not recommended. Furthermore, for the first time, our study has identified that Asian COVID-19 patients with comorbidities might benefit more from treatment with ACEIs, although the robustness of the evidence is weak. Together, our findings provide new insights into clinical strategies to improve the treatment and prognosis of patients with COVID-19.

\section{Acknowledgments}

Funding: None.

\section{Footnote}

Reporting Checklist: The authors have completed the PRISMA and MOOSE reporting checklist. Available at https://apm.amegroups.com/article/view/10.21037/apm$21-972 / \mathrm{rc}$

Conflicts of Interest: All authors have completed the ICMJE uniform disclosure form (available at https://apm. amegroups.com/article/view/10.21037/apm-21-972/coif). The authors have no conflicts of interest to declare.

Ethical Statement: The authors are accountable for all aspects of the work in ensuring that questions related to the accuracy or integrity of any part of the work are appropriately investigated and resolved.

Open Access Statement: This is an Open Access article distributed in accordance with the Creative Commons Attribution-NonCommercial-NoDerivs 4.0 International License (CC BY-NC-ND 4.0), which permits the noncommercial replication and distribution of the article with the strict proviso that no changes or edits are made and the original work is properly cited (including links to both the formal publication through the relevant DOI and the license). See: https://creativecommons.org/licenses/by-nc-nd/4.0/.

\section{References}

1. Han Q, Lin Q, Jin S, et al. Coronavirus 2019-nCoV: A brief perspective from the front line. J Infect 2020;80:373-7.

2. Zhou F, Yu T, Du R, et al. Clinical course and risk factors for mortality of adult inpatients with COVID-19 
in Wuhan, China: a retrospective cohort study. Lancet 2020;395:1054-62.

3. Wang X, Fang X, Cai Z, et al. Comorbid Chronic Diseases and Acute Organ Injuries Are Strongly Correlated with Disease Severity and Mortality among COVID-19 Patients: A Systemic Review and Meta-Analysis. Research (Wash D C) 2020;2020:2402961.

4. Fang L, Karakiulakis G, Roth M. Are patients with hypertension and diabetes mellitus at increased risk for COVID-19 infection? Lancet Respir Med 2020;8:e21.

5. Wan Y, Shang J, Graham R, et al. Receptor Recognition by the Novel Coronavirus from Wuhan: an Analysis Based on Decade-Long Structural Studies of SARS Coronavirus. J Virol 2020;94:e00127-20.

6. Li XC, Zhang J, Zhuo JL. The vasoprotective axes of the renin-angiotensin system: Physiological relevance and therapeutic implications in cardiovascular, hypertensive and kidney diseases. Pharmacol Res 2017;125:21-38.

7. Reynolds HR, Adhikari S, Pulgarin C, et al. ReninAngiotensin-Aldosterone System Inhibitors and Risk of Covid-19. N Engl J Med 2020;382:2441-8.

8. Mancia G, Rea F, Ludergnani M, et al. Renin-AngiotensinAldosterone System Blockers and the Risk of Covid-19. N Engl J Med 2020;382:2431-40.

9. Bean DM, Kraljevic Z, Searle T, et al. Angiotensinconverting enzyme inhibitors and angiotensin II receptor blockers are not associated with severe COVID-19 infection in a multi-site UK acute hospital trust. Eur J Heart Fail 2020;22:967-74.

10. Mehra MR, Desai SS, Kuy S, et al. Cardiovascular Disease, Drug Therapy, and Mortality in Covid-19. N Engl J Med 2020;382:e102.

11. Feng Z, Li J, Yao S, et al. The Use of Adjuvant Therapy in Preventing Progression to Severe Pneumonia in Patients with Coronavirus Disease 2019: A Multicenter Data Analysis. medRxiv 2020. doi: 10.1101/2020.04.08.20057539.

12. Macaskill P, Walter SD, Irwig L. A comparison of methods to detect publication bias in meta-analysis. Stat Med 2001;20:641-54.

13. Normand SL. Meta-analysis: formulating, evaluating, combining, and reporting. Stat Med 1999;18:321-59.

14. Yang G, Tan Z, Zhou L, et al. Effects of Angiotensin II Receptor Blockers and ACE (Angiotensin-Converting Enzyme) Inhibitors on Virus Infection, Inflammatory Status, and Clinical Outcomes in Patients With COVID-19 and Hypertension: A Single-Center Retrospective Study. Hypertension 2020;76:51-8.
15. Yan H, Valdes AM, Vijay A, et al. Role of Drugs Used for Chronic Disease Management on Susceptibility and Severity of COVID-19: A Large Case-Control Study. Clin Pharmacol Ther 2020;108:1185-94.

16. Zhang LK, Sun Y, Zeng H, et al. Calcium channel blocker amlodipine besylate therapy is associated with reduced case fatality rate of COVID-19 patients with hypertension. Cell Discov 2020;6:96.

17. Guo T, Fan Y, Chen M, et al. Cardiovascular Implications of Fatal Outcomes of Patients With Coronavirus Disease 2019 (COVID-19). JAMA Cardiol 2020;5:811-8.

18. Li J, Wang X, Chen J, et al. Association of ReninAngiotensin System Inhibitors With Severity or Risk of Death in Patients With Hypertension Hospitalized for Coronavirus Disease 2019 (COVID-19) Infection in Wuhan, China. JAMA Cardiol 2020;5:825-30.

19. Peng YD, Meng K, Guan HQ, et al. Zhonghua Xin Xue Guan Bing Za Zhi 2020;48:450-5.

20. Meng J, Xiao G, Zhang J, et al. Renin-angiotensin system inhibitors improve the clinical outcomes of COVID-19 patients with hypertension. Emerg Microbes Infect 2020;9:757-60.

21. Zhang P, Zhu L, Cai J, et al. Association of Inpatient Use of Angiotensin-Converting Enzyme Inhibitors and Angiotensin II Receptor Blockers With Mortality Among Patients With Hypertension Hospitalized With COVID-19. Circ Res 2020;126:1671-81.

22. Caldeira D, Alarcão J, Vaz-Carneiro A, et al. Risk of pneumonia associated with use of angiotensin converting enzyme inhibitors and angiotensin receptor blockers: systematic review and meta-analysis. BMJ 2012;345:e4260.

23. Imai Y, Kuba K, Penninger JM. The discovery of angiotensin-converting enzyme 2 and its role in acute lung injury in mice. Exp Physiol 2008;93:543-8.

24. Wawer MJ, Gray RH, Sewankambo NK, et al. Rates of HIV-1 transmission per coital act, by stage of HIV-1 infection, in Rakai, Uganda. J Infect Dis 2005;191:1403-9.

25. Yan R, Zhang Y, Li Y, et al. Structure of dimeric fulllength human ACE2 in complex with B0AT1. medRxiv 2020. doi: 10.1101/2020.02.17.951848.

26. Ye R, Liu Z. ACE2 exhibits protective effects against LPSinduced acute lung injury in mice by inhibiting the LPSTLR4 pathway. Exp Mol Pathol 2020;113:104350.

27. Tan WSD, Liao W, Zhou S, et al. Targeting the reninangiotensin system as novel therapeutic strategy for pulmonary diseases. Curr Opin Pharmacol 2018;40:9-17.

28. Clerkin KJ, Fried JA, Raikhelkar J, et al. COVID-19 and Cardiovascular Disease. Circulation 2020;141:1648-55. 
29. Grover A, Oberoi M. A systematic review and metaanalysis to evaluate the clinical outcomes in COVID-19 patients on angiotensin-converting enzyme inhibitors or angiotensin receptor blockers. Eur Heart J Cardiovasc Pharmacother 2021;7:148-57.

30. Strauss MH, Hall AS. Angiotensin receptor blockers may increase risk of myocardial infarction: unraveling the ARBMI paradox. Circulation 2006;114:838-54.

31. Cheng J, Zhang W, Zhang X, et al. Effect of angiotensinconverting enzyme inhibitors and angiotensin II receptor blockers on all-cause mortality, cardiovascular deaths, and cardiovascular events in patients with diabetes mellitus: a meta-analysis. JAMA Intern Med 2014;174:773-85.

32. Savarese G, Costanzo P, Cleland JG, et al. A meta-analysis reporting effects of angiotensin-converting enzyme inhibitors and angiotensin receptor blockers in patients

Cite this article as: Xie Q, Tang S, Li Y. The divergent protective effects of angiotensin-converting enzyme inhibitors and angiotensin receptor blockers on clinical outcomes of coronavirus disease 2019 (COVID-19): a systematic review and meta-analysis. Ann Palliat Med 2022;11(4):1253-1263. doi: 10.21037/apm-21-972 without heart failure. J Am Coll Cardiol 2013;61:131-42.

33. Ferrari R, Boersma E. The impact of ACE inhibition on all-cause and cardiovascular mortality in contemporary hypertension trials: a review. Expert Rev Cardiovasc Ther 2013;11:705-17.

34. Lancellotti P, Ancion A, D'Orio V, et al. Bradykinin and cardiovascular protection. Role of perindopril, an inhibitor of angiotensin conversion enzyme. Rev Med Liege 2018;73:197-205.

35. Liu Y, Huang F, Xu J, et al. Anti-hypertensive Angiotensin II receptor blockers associated to mitigation of disease severity in elderly COVID-19 patients. medRxiv 2020. doi: 10.1101/2020.03.20.20039586.

36. Zhao Y, Zhao Z, Wang Y, et al. Single-Cell RNA Expression Profiling of ACE2, the Receptor of SARSCoV-2. Am J Respir Crit Care Med 2020;202:756-9. 
Supplementary

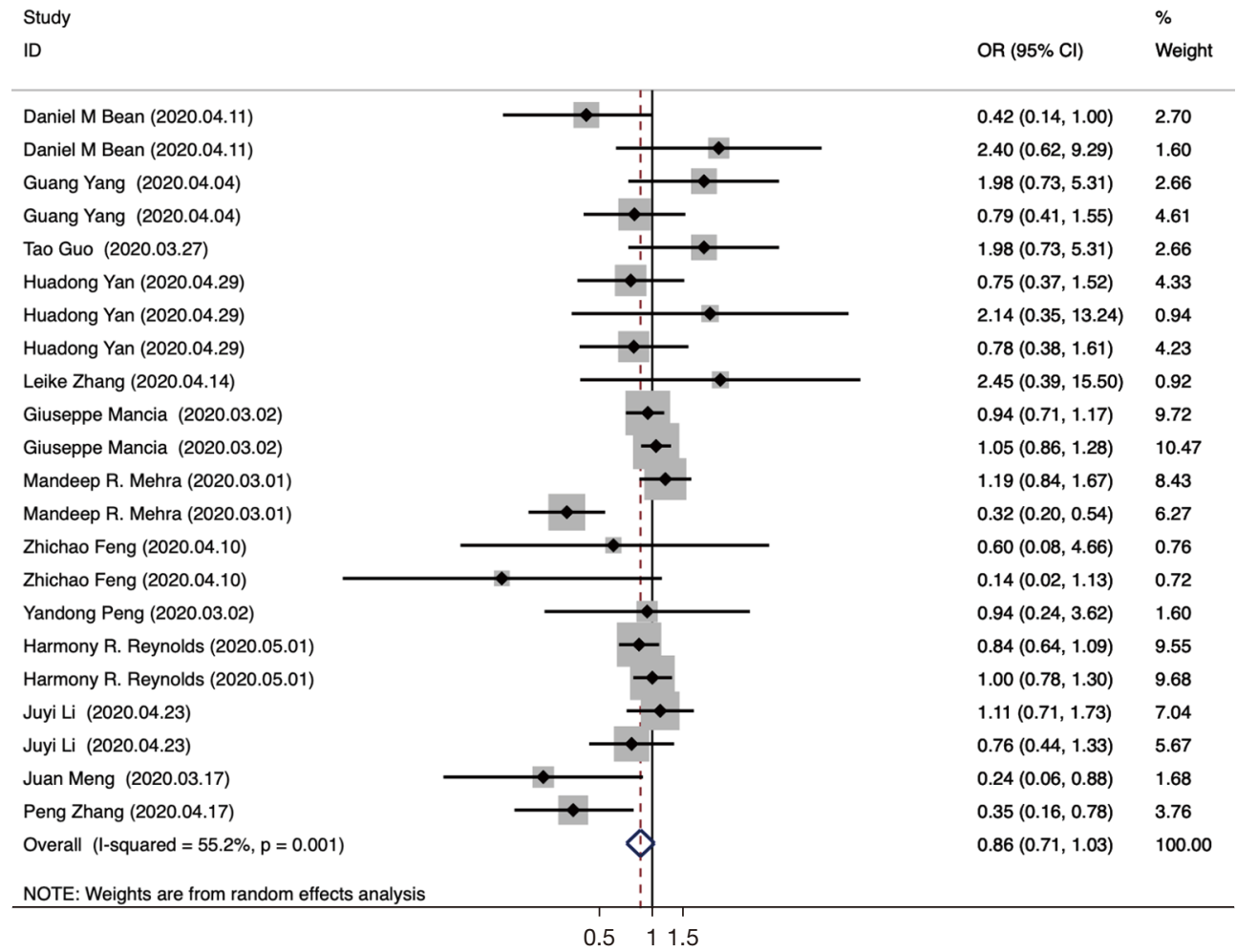

Figure S1 Forest plot showing the effect of medications on the risk of total and different clinical outcomes COVID-19-infected patients by random effects model analysis with crude OR values. In this and subsequent figures, the horizontal lines indicate the lower and upper limits of the $95 \%$ CI, the size of the gray squares reflects the relative weight of each study in the meta-analysis. COVID-19, coronavirus disease 2019; OR, odds ratio; CI, confidence interval. 
Study

ID

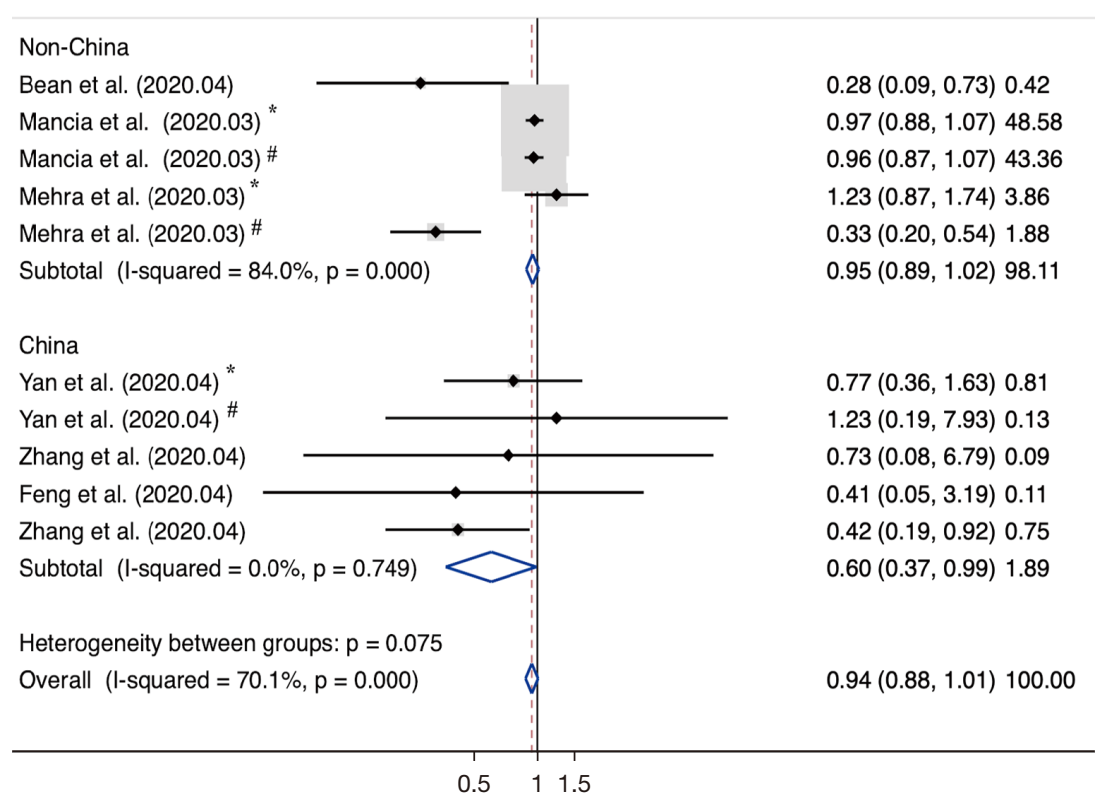

Figure S2 Forest plot showing the effect of study locations on the risk of clinical outcomes SARS-CoV-2-infected patients depending on adjust OR values using fixed effects model analysis. OR, odds ratio; CI, confidence interval; SARS-CoV-2, severe acute respiratory syndrome coronavirus 2 . 


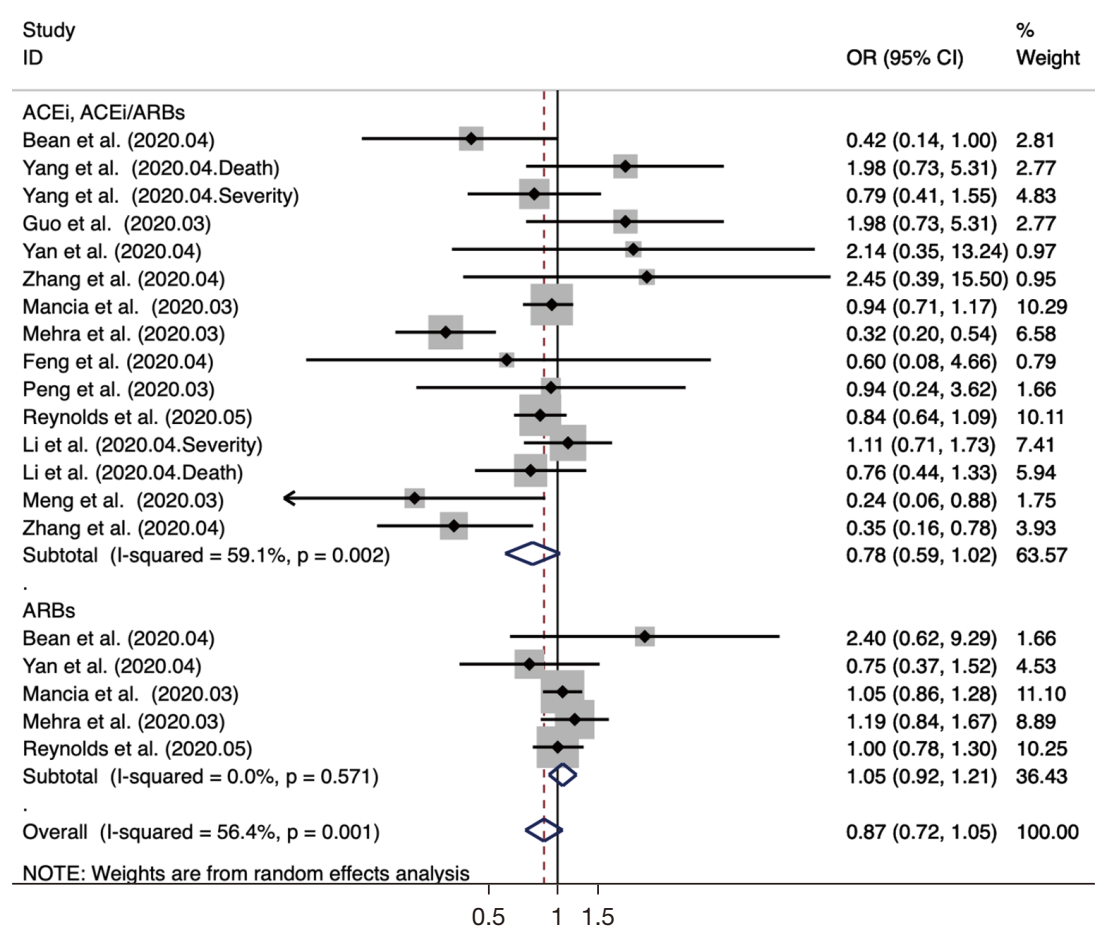

Figure S3 Forest plot showing the effect of different medications on the risk of COVID-19 patients' clinical outcomes by random effects model analysis with crude OR values. OR, odds ratio; CI, confidence interval; ACEI, angiotensin-converting enzyme inhibitor; ARBs, angiotensin receptor blockers; COVID-19, coronavirus disease 2019.

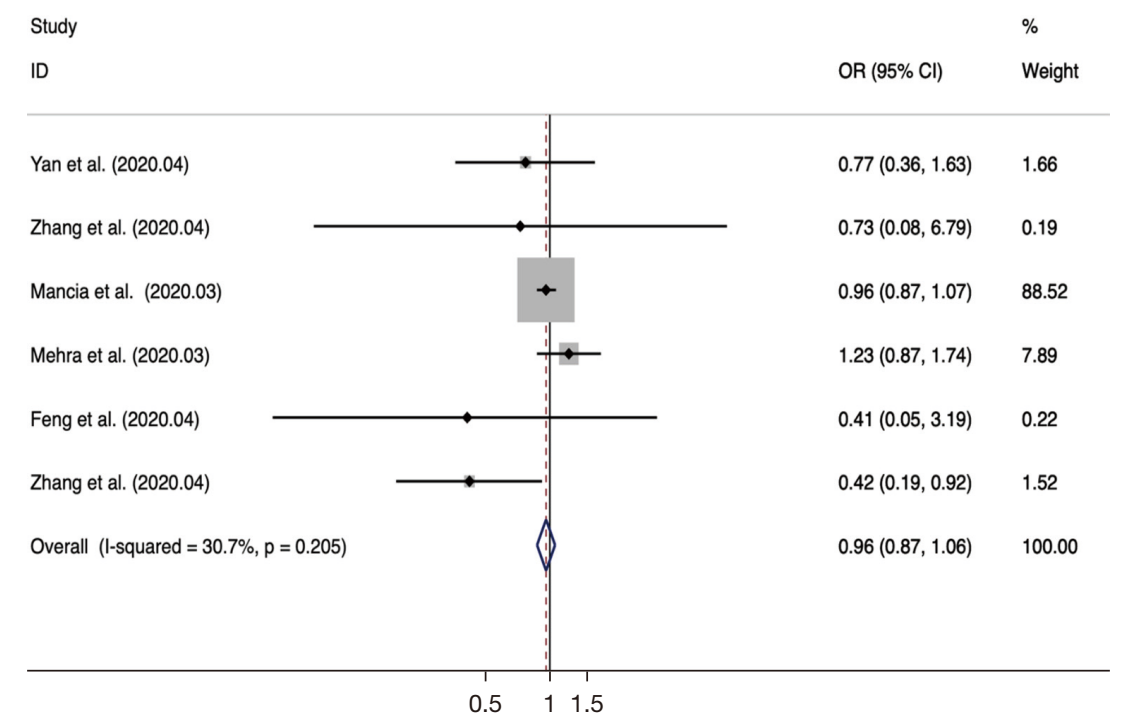

Figure S4 Forest plot showing the effect of ARBs, ACEI/ARBs medications on the risk of clinical outcomes SARS-CoV-2-infected patients depending on adjust OR values using random effects model analysis by fixed effects modle analysis. OR, odds ratio; CI, confidence interval; ARBs, angiotensin receptor blockers; ACEI, angiotensin-converting enzyme inhibitor; SARS-CoV-2, severe acute respiratory syndrome coronavirus 2 . 


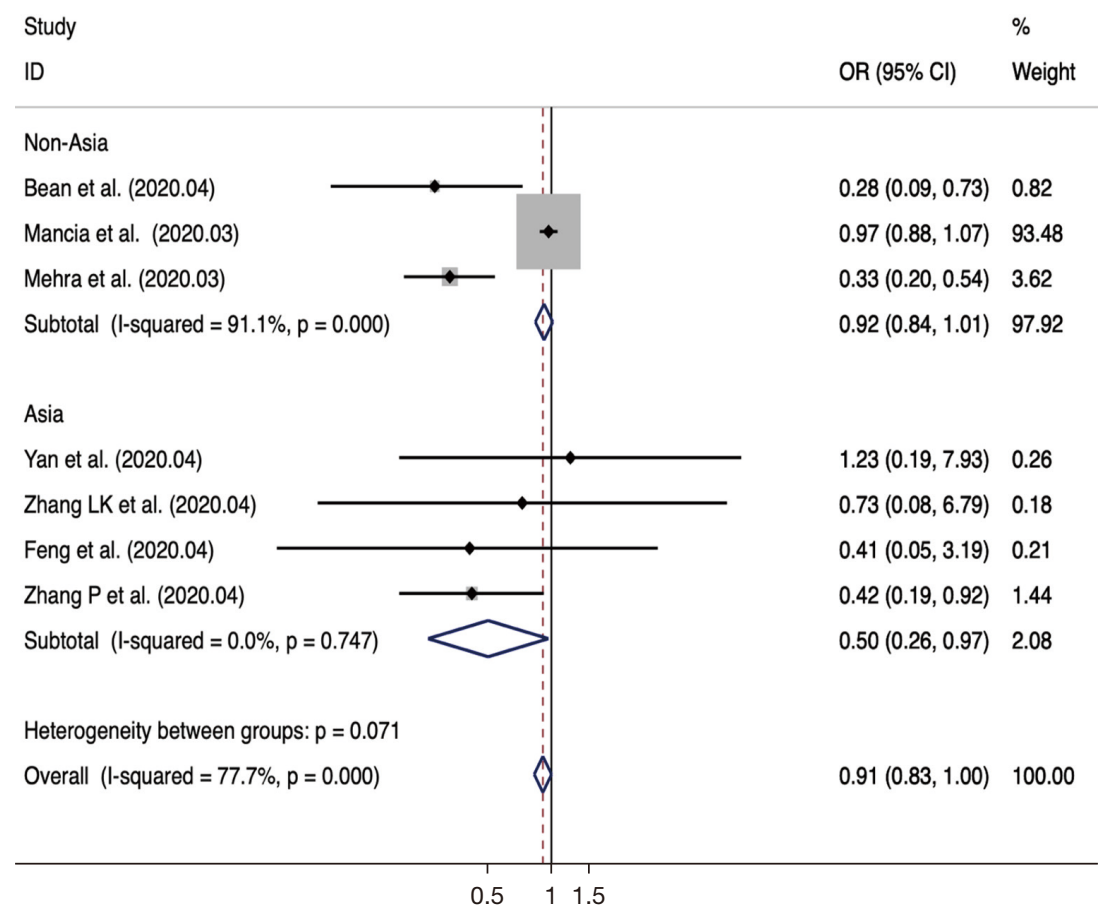

Figure S5 Forest plot showing the effect of ACEI, ACEI/ARBs medication on the risk of clinical outcomes SARS-CoV-2-infected patients depending on adjust OR values using random effects model analysis. OR, odds ratio; CI, confidence interval; ACEI, angiotensin-converting enzyme inhibitor; ARBs, angiotensin receptor blockers; SARS-CoV-2, severe acute respiratory syndrome coronavirus 2. 
Table S1 Risk of bias in studies providing relative risk estimates

\begin{tabular}{|c|c|c|c|c|c|}
\hline First author & \multicolumn{4}{|r|}{ Adjusted confounders } & Risk of bias \\
\hline Bean & 2020 & Yes & Yes & Hypertension, diabetes mellitus, ischaemic heart disease, heart failure & Low risk \\
\hline Yang & 2020 & Yes & Yes & None & Moderate risk \\
\hline Guo & 2020 & None & None & None & High risk \\
\hline Zhang & 2020 & Yes & Yes & $\begin{array}{l}\text { The delay from symptom onset to hospital admission, and therapies } \\
\text { administration }\end{array}$ & Low risk \\
\hline Mancia & 2020 & Yes & Yes & Drugs and coexisting conditions & Low risk \\
\hline Mehra & 2020 & Yes & Yes & None & Moderate risk \\
\hline $\mathrm{Li}$ & 2020 & Yes & Yes & None & Moderate risk \\
\hline Meng & 2020 & None & None & None & High risk \\
\hline Zhang & 2020 & Yes & Yes & Conditions & Low risk \\
\hline
\end{tabular}

Risk of bias assessed by level of adjustment. Low risk, adjustment of age, sex, and at least one other covariate; Moderate risk, adjustment of at least age and sex; High risk, no adjustment. BMI, body mass index.

Table S2 Sensitivity analyses to evaluate the contribution of each study to the pooled estimation by excluding each of the studies one after the others

\begin{tabular}{lc}
\hline Study author & Atrial fibrillation OR with $95 \% \mathrm{Cl}$ after removing the study \\
\hline Bean & $0.83(0.68-1.02)$ \\
Yang & $0.79(0.62-0.99)$ \\
Yan & $0.78(0.62-0.98)$ \\
Zhang & $0.79(0.63-0.98)$ \\
Mancia & $0.65(0.44-0.97)$ \\
& $0.65(0.44-0.97)$ \\
Mehra & $0.72(0.57-0.92)$ \\
& $0.94(0.82-1.08)$ \\
Feng & $0.79(0.64-0.99)$ \\
Zhang & $0.83(0.67-1.02)$ \\
\hline
\end{tabular}

OR, odds ratio; $\mathrm{Cl}$, confidence interval. 
Table S3 Meta regression estimate outcomes of between-study variance

\begin{tabular}{|c|c|c|c|c|c|c|c|}
\hline$\frac{\text { Group }}{\text { By crude OR }}$ & $\begin{array}{l}\text { Covariates } \\
\text { Study location }\end{array}$ & $\begin{array}{c}\operatorname{Exp}(\mathrm{B}) \\
-0.073\end{array}$ & $\frac{\text { Std. Err }}{0.143}$ & $\frac{t}{-0.51}$ & $\frac{P \text { value }}{0.620}$ & \multicolumn{2}{|c|}{$95 \% \mathrm{Cl}$} \\
\hline & Journal & -0.458 & 0.748 & -0.61 & 0.549 & -2.036 & 1.121 \\
\hline & Medications & 0.177 & 0.216 & 0.82 & 0.424 & -0.278 & 0.631 \\
\hline & Article risk & -0.150 & 0.298 & -0.50 & 0.622 & -0.779 & 0.479 \\
\hline \multirow[t]{4}{*}{ By adjust OR } & Study location & -0.057 & 0.289 & -0.20 & 0.85 & -0.763 & 0.845 \\
\hline & Journal & -0.353 & 0.953 & -0.37 & 0.724 & -2.685 & 1.980 \\
\hline & Medications & 0.052 & 0.324 & 0.16 & 0.877 & -0.740 & 0.845 \\
\hline & Article risk & -0.353 & 0.953 & -0.37 & 0.724 & -2.685 & 1.980 \\
\hline
\end{tabular}

Exp (B), exponential (B); Std. Err, standard error; Cl, confidence interval; OR, odds ratio.

Table S4 Egger's test for small-study effects

\begin{tabular}{lllcccc}
\hline Group & Std_Eff & Coef. & Std. Err. & $t$ & P value & $95 \%$ Cl \\
\hline By crude OR & Slope & 0.019 & 0.124 & 0.15 & 0.883 & -0.240 \\
& Bias & -0.539 & 0.532 & -1.01 & 0.324 & -1.649 \\
\multirow{2}{*}{ By adjust OR } & Slope & 0.025 & 0.074 & 0.35 & 0.735 & -0.145 \\
& Bias & -1.222 & 0.673 & -1.82 & 0.107 & -2.775 \\
\hline
\end{tabular}

Std_Eff, standard effects; Coef., coefficient; Std. Err, standard error; Cl, confidence interval; OR, odds ratio.

Table S5 Begg's test for small-study effects

\begin{tabular}{|c|c|c|c|c|c|c|c|}
\hline Group & $\begin{array}{l}\text { Adj. Kendall's } \\
\text { score }\end{array}$ & $\begin{array}{l}\text { Std. Dev. of } \\
\text { score }\end{array}$ & $\begin{array}{l}\text { Number of } \\
\text { studies }\end{array}$ & $z$ & $P$ value & $\begin{array}{l}\text { z continuity } \\
\text { corrected }\end{array}$ & $\begin{array}{l}\text { P continuity } \\
\text { corrected }\end{array}$ \\
\hline By crude OR & -8 & 35.46 & 22 & -0.23 & 0.822 & 0.20 & 0.844 \\
\hline By adjust OR & -11 & 11.18 & 10 & -0.98 & 0.325 & 0.89 & 0.371 \\
\hline
\end{tabular}

Adj., adjust; Std. Dev., standard deviation; OR, odds ratio. 


\section{Appendix: search criteria}

\section{PubMed}

\#1. $(((((((((C O V I D 19[$ Title/Abstract]) OR COVID-19[Title/Abstract] $)$ OR 2019 novel coronavirus disease[Title/Abstract]) OR SARS-CoV-2[Title/Abstract]) OR COVID-19 virus disease[Title/Abstract]) OR 2019 novel coronavirus infection[Title/ Abstract]) OR 2019-nCoV infection[Title/Abstract]) OR 2019-nCoV infection[Title/Abstract]) OR coronavirus disease 2019[Title/Abstract]) OR 2019-nCoV disease[Title/Abstract]) OR COVID-19 virus infection[Title/Abstract]

\#2. (Renin-angiotensin-aldosterone System[Title/Abstract]) OR RAAS[Title/Abstract]

\#3. (((angiotensin converting enzyme inhibitors[Title/Abstract]) OR ACEIs[Title/Abstract]) OR ACEis[Title/Abstract]) OR ACEi[Title/Abstract]

\#4. ((angiotensin receptor blockers[Title/Abstract]) OR ARBs[Title/Abstract]) OR ARB[Title/Abstract]

\#5. \#3 OR \#4

\#6. \#2 OR \#5

\#7. \#1 AND \#6

\section{Embase}

1. COVID19. ab. ti.

2. SARS-CoV-2. ab. ti.

3. 2019-nCoV disease. ab. ti.

4. 2019-nCoV. ab. ti.

5. coronavirus disease 2019. ab. ti.

6. 1 or 2 or 3 or 4 or 5 or

7. Renin-angiotensin-aldosterone System. ab. ti.

8. RAAS. ab. ti.

9. angiotensin converting enzyme inhibitors. ab. ti.

10. ACEIs. ab. ti.

11. ACEis. ab. ti.

12. ACEi. ab. ti.

13. angiotensin receptor blockers. ab. ti.

14. ARBs. ab. ti.

15. ARB. ab. ti.

16.7 or 8

17. 9 or 10 or 11 or 12

18. 13 or 14 or 15

19. 16 or 17 or 18

20.6 and 19 